\title{
DDX3 directly facilitates IKK $\alpha$ activation and regulates downstream signalling pathways
}

\author{
Anthony Fullam, Lili Gu, Yvette Höhn and Martina Schröder \\ Department of Biology, Institute of Immunology, Maynooth University, Maynooth, Co. Kildare, Ireland \\ Correspondence: Martina Schröder (martina.schroeder@mu.ie)
}

Received: 27 February 2018 Revised: 18 October 2018 Accepted: 19 0ctober 2018

Accepted Manuscript online: 19 October 2018 Version of Record published: 20 November 2018
DDX3 is a DEAD-box RNA helicase that we and others have previously implicated in antiviral immune signalling pathways leading to type I interferon (IFN) induction. We previously demonstrated that it directly interacts with the kinase IKK it activation, and then facilitates phosphorylation of the transcription factor IRF3 by IKKe. However, the TLR7/9 (Toll-like receptor 7/9)-mediated pathway, one of the most physiologically relevant IFN induction pathways, proceeds independently of IKK $\varepsilon$ or the related kinase TBK1 (TANK-binding kinase 1). This pathway induces type I IFN production via the kinases NIK (NF-KB-inducing kinase) and IKK $\alpha$ and is activated when plasmacytoid dendritic cells sense viral nucleic acids. In the present study, we demonstrate that DDX3 also directly interacts with $\mathrm{IKK} \alpha$ and enhances its autophosphorylation and -activation. Modulation of DDX3 expression consequently affected NIK/IKK $\alpha$-mediated IRF7 phosphorylation and induction of type I interferons. In addition, alternative NF- $\mathrm{KB}$ (nuclear factor- $\kappa \mathrm{B}$ ) activation, another pathway regulated by NIK and IKK $\alpha$, was also down-regulated in DDX3 knockdown cells. This substantially broadens the effects of DDX3 in innate immune signalling to pathways beyond TBK1/IKK $\varepsilon$ and IFN induction. Dysregulation of these pathways is involved in disease states, and thus, our research might implicate DDX3 as a potential target for their therapeutic manipulation.

\section{Introduction}

Several DExD/H-box RNA helicases, including DDX3, have recently been implicated to participate in innate antiviral responses mediated by pattern recognition receptors (PRRs), either by directly acting as receptors for viral nucleic acids or by functioning as downstream signalling intermediates [1]. Our previous work has shown that DDX3 can act as a multifunctional adaptor molecule in the RIG-I (retinoic acid-inducible gene I) signalling pathway [2-4]: it directly interacts with the kinase IKKe (IKB kinase $\varepsilon$ ) and enhances its autophosphorylation and -activation. IKKe also directly phosphorylates DDX3 at several serine residues in its N-terminal tail region, with phosphorylation of serine 102 being required for the subsequent recruitment of its substrate IRF3 (interferon regulatory factor 3) into the complex and subsequent induction of type I interferons (IFNs) [3]. Our data therefore suggested that DDX3 acts as a signalling adaptor molecule that facilitates the co-ordinated activation of IKKE and its substrate IRF3 [3]. Other groups have confirmed the role of DDX3 in IFN induction, but have placed it at different levels in the signalling pathways [5-9]: it was suggested that DDX3 can directly bind to the ifnb promoter and act as a transcriptional regulator [9], and also that DDX3 acts as a receptor for HCV RNA and is placed upstream of MAVS (mitochondrial antiviral signalling) [7], the crucial mitochondrial adaptor molecule in the RIG-I pathway.

While most innate immune signalling pathways leading to type I interferon induction, including those mediated by Toll-like receptor (TLR) 3, TLR4, the RIG-like helicases (RLHs) and cytosolic DNA receptors, converge on IKKe/TBK1 (TANK-binding kinase 1) for phosphorylation of IRF3 and IRF7, it was shown that TLR7 and TLR9 signalling to IFN induction is independent of these two kinases $[10,11]$. TLR7 and TLR9 detect viral nucleic acids (single-stranded RNA and unmethylated 
CpG DNA, respectively) in the endosomal lumen and trigger the induction of large amounts of type I interferons, in particular IFN- $\alpha$ [12]. This pathway is mainly active in plasmacytoid dendritic cells due to their expression of TLR7 and TLR9 and high constitutive levels of the transcription factor IRF7. IRF7 activation in this pathway is mediated by NIK (NF-KB-inducing kinase) and IKK $\alpha$ [11]. NIK facilitates IKK $\alpha$ activation downstream of Myd88 and TRAF6 (TNF receptor-associated factor 6), conferring on it the ability to phosphorylate IRF7 and activate type I interferon promoters [13,14]. TLR7/9-mediated IFN production from plasmacytoid dendritic cells (pDCs) is thought to be highly relevant during viral infections [15], and thus, we investigated here whether DDX3 also contributes to this physiologically important IKKe/TBK1-independent IFN induction pathway.

In addition, NIK and IKK $\alpha$ also co-operate to activate the alternative NF- $\kappa B$ (nuclear factor- $\kappa \mathrm{B}$ ) pathway, which leads to processing of p100 to the active p52 NF- $\kappa B$ subunit [16]. This pathway is mainly activated downstream of CD40 and related receptors, and has been shown to play a role in the development of secondary lymphoid organs, bone metabolism, survival and maturation of B-cells and thymic deletion of autoimmune T-cells. Hyperactivation of the p52 pathway has been linked to cellular transformation and the emergence of various haematopoietic and solid tumours [17]. It has also been shown that the alternative NF- $\kappa \mathrm{B}$ pathway is activated downstream of RIG-I, where it contributes to the induction of chemokines and antiviral immune responses $[18,19]$.

Thus, IKK $\alpha$ is of major importance for these two immunorelevant signalling pathways, and we therefore aimed to investigate whether DDX3 regulates IKK $\alpha$ activation in a similar manner to IKKe [3]. The data we present here demonstrate that DDX3 can indeed directly enhance IKK $\alpha$ activation and that it is a positive regulator of NIK-IKK $\alpha$-mediated IRF7 activation and the alternative NF- $\kappa B$ pathway. This further broadens the involvement of DDX3 in innate immune signalling pathways.

\section{Experimental}

\section{Plasmids, recombinant proteins and antibodies}

DDX3 and K7 expression constructs (in pCMV-myc, pCMV-Ha or pHisParallel2) have been described in our previous studies [3,4]. IKK $\alpha$-Flag and NIK-Flag were provided by Prof. Luke O’Neill (Trinity College Dublin), and Flag-IRF7 was provided by Prof. Kate Fitzgerald (University of Massachusetts). GST (glutathione-S-transferase)-IRF7 (aa 468-503) peptides and constructs were kindly provided by Prof. John Hiscott and Dr Qiang Sun (McGill University, Montreal). Purified recombinant protein kinase GST-IKK $\alpha$ was purchased from Millipore and recombinant GST-NIK was purchased from Proqinase (Freiburg, Germany). The antibodies used were anti-Flag-M2 mAb (Sigma-Aldrich), anti-Myc mAb clone 9E10 (Sigma-Aldrich), anti-HA (hemagglutinin) mAb (Covance), anti-DDX3 (Santa Cruz or Bethyl Laboratories), anti-p52/p100 (Millipore), anti-IRF7 (Santa Cruz), anti-IKK $\alpha$, anti-phospho-Ser176/180-IKK $\alpha$, anti-phospho-Ser 866/ 870-p100, and anti-phospho-Ser471/472-IRF7 (all Cell Signaling Technology), anti-His (Sigma-Aldrich), and anti-GST (Promega). Secondary AlexaFluor488- and AlexaFluor594-coupled antibodies were purchased from Invivogen.

\section{Cell culture and transfection}

HEK293T and HEK293_TLR7 cells (Invivogen) were maintained in DMEM, and THP1 cells were maintained in RPMI. Media were supplemented with Glutamax ${ }^{\mathrm{TM}}, 10 \%$ foetal calf serum, and gentamycin. Transient DNA transfections for immunoprecipitations and pulldowns were performed using the calcium phosphate method. The total amount of DNA was kept constant by the addition of empty vector. Transfection for reporter gene assays was performed in 96-well plate format using Genejuice ${ }^{\mathrm{TM}}$ with a total of $230 \mathrm{ng}$ of DNA.

\section{Short hairpin RNA knockdown of DDX3}

Lentiviral particles were generated by transfecting HEK293T cells with either pTRIPZ shDDX3 (V2THS_228965, Thermo Scientific) or the corresponding non-silencing control (NSC), and the packaging vectors $\mathrm{pSDAX} 2$ and $\mathrm{pMD} 2$ in the presence of chloroquine sulfate. Lentivirus-containing supernatants were harvested and concentrated using PEG precipitation. HEK293, HEK293_TLR7, or THP1 cells were transduced with shDDX3 or NSC lentivirus at an MOI of 10. Transduced cells were selected in medium containing $3 \mu \mathrm{g} /$ $\mathrm{ml}$ puromycin. For use in experiments, cells were cultured in the absence of puromycin and DDX3 knockdown 
was induced by the addition of $0.5 \mu \mathrm{g} / \mathrm{ml}$ doxycycline for $24-48 \mathrm{~h}$. Successful knockdown of DDX3 was confirmed by real-time PCR and Western blot (WB) analysis.

\section{His-pulldown assays}

His-tagged DDX3 was purified from Escherichia coli BL21(DE3) cultures transformed with relevant expression constructs using Nickel-Agarose beads. For pulldowns, equal amounts of the different His-tagged DDX3 truncations were used, as estimated by SDS-PAGE and Coomassie staining prior to use. Cell lysates containing Flag-tagged protein expressed in HEK293T cells, or $0.1 \mu \mathrm{g}$ of GST-IKK $\alpha$ (Millipore) or GST-NIK (Proqinase), were then incubated with His-tagged DDX3 immobilised on Nickel-Agarose. Protein complexes were precipitated and washed thoroughly, before being subjected to SDS-PAGE and WB analysis.

\section{Co-immunoprecipitation assays}

Co-immunoprecipitation assays were performed from cell lysates of transiently transfected HEK293T or HEK293_TLR7 cells, which were harvested $24 \mathrm{~h}$ after transfection. Cells were lysed in IP (immunoprecipitation) lysis buffer $(50 \mathrm{mM}$ Hepes $\mathrm{pH} 7.4,150 \mathrm{mM} \mathrm{NaCl}, 2 \mathrm{mM}$ EDTA, 1\% NP-40, $10 \%$ glycerol, $10 \mathrm{mM} \mathrm{NaF}, 1 \%$ Triton X-100, $10 \mathrm{mM}$ DTT, and protease inhibitors). Cell lysates were incubated with $20 \mu \mathrm{l}$ of Flag-M2 Agarose (Sigma-Aldrich) or Protein G agarose that had been pre-coupled with relevant antibodies at $4^{\circ} \mathrm{C}$ overnight and blocked with $5 \%$ bovine serum albumin. The immunoprecipitated protein complexes were washed thoroughly and then eluted by boiling in Laemmli sample buffer, before being subjected to SDS-PAGE and WB analysis. Endogenous co-immunoprecipitation between DDX3and IKK $\alpha$ was carried out from lysates of CLO75-stimulated HEK293_TLR7, and a matched isotype control antibody was used as a control for nonspecific pulldown.

\section{Immunofluorescent staining and confocal microscopy}

HEK293 cells were seeded on sterile glass cover slips in six-well plates and transfected with expression constructs as indicated using the calcium phosphate method. After $24 \mathrm{~h}$, they were washed, fixed with $4 \%$ paraformaldehyde, and permeabilised with $0.5 \%$ Triton X-100. After blocking with BSA, cells were stained with the appropriate primary antibodies, followed by Alexa-Fluor488- or AlexaFluor594-labelled anti-species secondary antibody (Invitrogen). After staining, cells were mounted in SlowFade Gold Antifade Reagent containing DAPI (Invitrogen) on microscope slides and sealed using clear nail varnish. Cells were viewed using an Olympus Fluoview Confocal Microscope, and analysed using the Olympus Fluoview FV10-ASW software. Cells in multiple different frames were examined and recorded in each of two repeat experiments. The determination of Pearson's correlation coefficients (PCCs) was carried out using the Fluoview software on transfected cells.

\section{In vitro kinase assays}

In vitro kinase assays were carried out with $55 \mathrm{ng}$ of recombinant GST-IKK $\alpha$ (Proqinase). This was incubated with purified recombinant substrates in kinase assay buffer (50 mM Hepes, $\mathrm{pH} 7.5,5 \mathrm{mM}$ glycerophosphate, $2 \mathrm{mM}$ dithiothreitol, $0.1 \mathrm{mM} \mathrm{Na}_{3} \mathrm{VO}_{4}$, and $10 \mathrm{mM} \mathrm{MgCl}$ ) in the presence of $20 \mu \mathrm{M}$ unlabelled ATP for $30 \mathrm{~min}$ at $30^{\circ} \mathrm{C}$. Samples were analysed by SDS-PAGE and WB analysis with phospho-specific antibodies. Alternatively, kinase assays were carried out in the presence of $10 \mu \mathrm{M}$ unlabelled ATP and $5 \mu \mathrm{Ci}$ of $\left[{ }^{\gamma} 32 \mathrm{P}\right]-$ ATP. Samples were then analysed by SDS-PAGE and autoradiography.

\section{Reporter gene assays}

Ifnb or Ifna4 reporter assays were carried out in HEK293 or HEK293_TLR7 cells in 96-well plate format. Cells were transfected with reporter constructs and expression vectors using GeneJuice (Merck/Millipore). About $60 \mathrm{ng}$ of ifn promoter Firefly luciferase reporter gene construct was used in conjunction with $20 \mathrm{ng}$ of a pGL3-Renilla luciferase construct (ifnb reporter and pGL3-Renilla provided by Andrew Bowie, Trinity College Dublin, ifna4 reporter provided by Pamela Osterlund, National Public Health Institute Finland). The total amount of DNA transfected was kept constant at $230 \mathrm{ng} /$ well by the addition of matching empty vector DNA. Firefly and Renilla Luciferase activity was measured $24 \mathrm{~h}$ after transfection. Renilla Luciferase readings were used to normalise for transfection efficiency. 


\section{Real-time PCR analysis}

RNA was isolated from stimulated and unstimulated THP1 NSC and shDDX3 cells using a Macherey Nagel RNA isolation kit according to manufacturer's instructions. cDNA was synthesised using iScript Reverse Transcriptase (Bio-Rad), and cDNA concentration was then adjusted to $200 \mathrm{ng} / \mu \mathrm{l}$. Real-time PCR analysis was conducted using Evagreen ${ }^{\circledR}$ Mastermix (Metabion) according to manufacturer's instructions, using an annealing temperature of $57^{\circ} \mathrm{C}$ for IFN $\beta$ and $60^{\circ} \mathrm{C}$ for BAFF, HPRT, and DDX3 primers. Primer sequences are available upon request. Relative expression levels were calculated using the $\Delta \Delta C_{\mathrm{t}}$ method with HPRT as the housekeeping gene and unstimulated NSC cells as the reference. DDX3 knockdown of between 60 and $90 \%$ was achieved in individual experiments.

\section{HEK-Blue TM IFN assay}

Supernatants were collected from CL075-stimulated THP1 cells and added to HEK-Blue IFN $\alpha / \beta$ sensor cells (Invivogen) plated in 96-wps. SEAP production from the exposed reporter cells was then measured using QUANTI-Blue ${ }^{\mathrm{TM}}$ reagent. SEAP production correlates to IFN $\alpha / \beta$ levels in the supernatant.

\section{Data representation and statistical analysis}

All data shown are representative of at least two independent repeat experiments, most are representative of three to five repeat experiments. Luciferase reporter gene assays are representative of at least three independent repeat experiments. Statistical analysis was carried out using a paired Student's $t$-test unless indicated otherwise. For clear representation, WBs have been cropped. Where a black box has been placed around WB panels, this indicates that they were taken from the same original autorad exposure and can thus be compared for intensity. Any adjustments for brightness and contrast that have been applied have been applied evenly across the whole (boxed) panel.

\section{Results}

\section{DDX3 interacts directly with IKKa}

We first tested whether DDX3 and IKK $\alpha$ physically interact. Initially, we overexpressed Flag-tagged IKK $\alpha$ and myc-tagged DDX3 in HEK293T cells and immunoprecipitated IKK $\alpha$ with a Flag antibody. DDX3 clearly co-immunoprecipitated with Flag-IKK $\alpha$, but was not pulled down by the Flag antibody in the absence of Flag-IKK $\alpha$ (Figure 1a). In this experiment, we also observed that overexpression of IKK $\alpha$ induced a higher-molecular-weight form of DDX3 (Figure 1a, middle panel) that likely represents a phosphorylated form of DDX3. We next carried out a similar co-immunoprecipitation experiment with overexpressed Flag-tagged NIK and myc-tagged DDX3 to determine whether DDX3 also interacts with NIK, the kinase that can activate IKK $\alpha$ in the alternative NF- $\kappa B$ pathway and downstream of TLR7, 8, and 9. DDX3 clearly co-immunoprecipitated with Flag-NIK, but was not pulled down by the Flag antibody in the absence of Flag-NIK (Figure 1b). Endogenous IKK $\alpha$ also strongly co-immunoprecipitated with NIK (Figure 1b, bottom panel), meaning that the observed interaction between DDX3 and NIK could be indirect and bridged by IKK $\alpha$. Next, we therefore conducted pulldown experiments with purified recombinant proteins. Recombinant His-tagged DDX3 purified from E. coli was used in pulldowns with recombinant GST-tagged IKK $\alpha$. In these pulldowns, we observed direct binding of GST-IKK $\alpha$ to His-tagged DDX3 coupled to Ni-Agarose (Ni-Ag) beads (Figure 1c). IKK $\alpha$ did not bind to empty Ni-Ag beads that were used as a control. This suggests that DDX3 3 and IKK $\alpha$ can directly interact with each other. Similar pulldown experiments with purified GST-NIK were inconclusive, and thus, we are unable to conclude whether DDX3 and NIK also directly interact or whether the interaction is bridged by other proteins, such as IKK $\alpha$. In addition to these co-immunoprecipitation and pulldown experiments, we also visualised the cellular localisation of co-expressed myc-DDX3 and Flag-IKK $\alpha /$ NIK using immunofluorescent staining and confocal microscopy. DDX3 and IKK $\alpha$ co-localised in the cytoplasm of transfected cells with an average PCC of 0.63 . When Flag-NIK was also co-expressed, the staining pattern or PCC did not change significantly (Supplementary Figure S1).

We next mapped the interaction between DDX3 and IKK $\alpha$, using a range of His-tagged truncation mutants of DDX3. IKK $\alpha$ interacted with full-length DDX3, DDX3 (aa 1-408) and DDX3 (aa 139-408), but not with DDX3 (aa 409-662) or DDX3 (aa 172-408) (Figure 1d). This suggests that IKK $\alpha$ interacts with a region between aa 139 and 172 of DDX3. Interestingly, this means that the main interaction site for IKK $\alpha$ in DDX3 
a

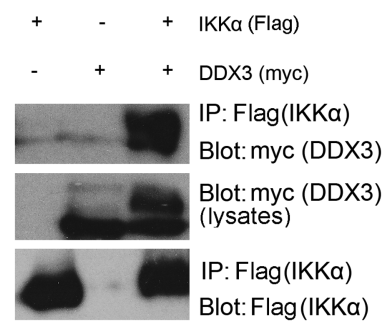

c

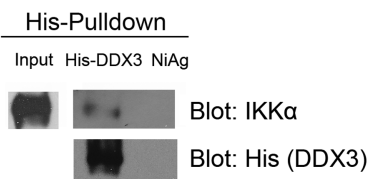

b

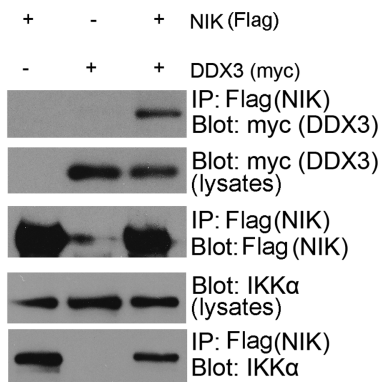

d

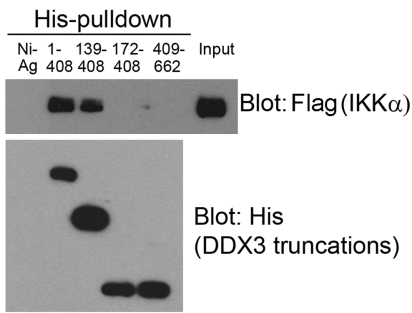

e
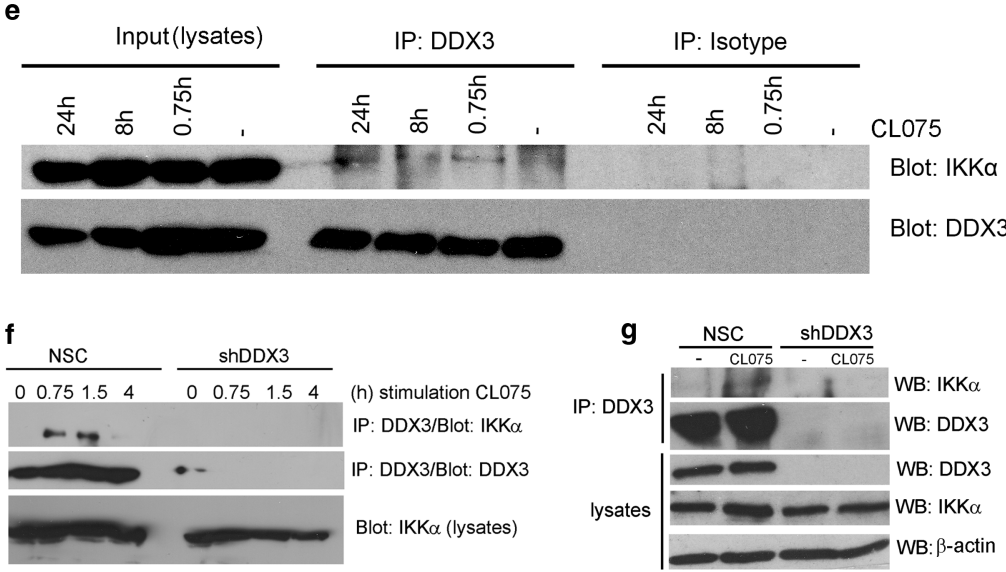

Figure 1. DDX3 interacts directly with IKKa following stimulation of the TLR7 pathway.

(a) HEK293T cells were transfected with expression constructs for Flag-IKK $\alpha$ and myc-DDX3. Cell lysates were harvested, lysed, and subjected to IP with anti-Flag antibodies. Washed immunoprecipitates and corresponding cell lysates were analysed by SDS-PAGE and Western blotting with the indicated antibodies to reveal co-immunoprecipitation of myc-DDX3. (b) Similar to (a), but cells were transfected with an expression construct for Flag-NIK instead of Flag-IKK $\alpha$ to reveal co-immunoprecipitation of myc-DDX3 with Flag-NIK. Samples were also probed for co-immunoprecipitation of endogenous IKK $\alpha$ (bottom panel).

(c) Purified recombinant His-DDX3 coupled to Nickel-Agarose ( $\mathrm{Ni}-\mathrm{Ag}$ ) beads was incubated with recombinant GST-IKK $\alpha$ (Millipore). Empty Ni-Ag beads were used as a control for non-specific binding. Beads were washed, boiled, and supernatants were subjected to SDS-PAGE and Western blotting alongside an input sample, to show direct binding of GST-IKK $\alpha$ to His-DDX3. (d) To map the DDX3-IKK $\alpha$ interaction, recombinant His-DDX3 truncation proteins were coupled to Ni-Ag beads and incubated with a lysate from HEK293T cells expressing Flag-IKK $\alpha$ (amino acid positions of truncated DDX3 indicated in the figure). Empty $\mathrm{Ni}-\mathrm{Ag}$ beads were used as a control for non-specific binding. Beads were washed, boiled, and supernatants were subjected to SDS-PAGE and Western blotting alongside an input sample. (e) HEK293_TLR7 cells were stimulated for the indicated time periods with $1 \mu \mathrm{g} / \mathrm{ml}$ CL075 (a TLR7/8 ligand). Cell lysates were subjected to IP with an antibody against endogenous DDX3 or a corresponding isotype control antibody, followed by SDS-PAGE and WB analysis to reveal co-immunoprecipitation of endogenous IKK $\alpha$. (f) HEK293_TLR7 cells stably transduced with either an inducible shDDX3 or a corresponding NSC plasmid were treated with doxycycline for $48 \mathrm{~h}$ to induce shRNA expression and then stimulated for the indicated time periods with the TLR7/8 ligand CL075. Cell lysates were subjected to IP with an antibody against endogenous DDX3, followed by SDS-PAGE and WB analysis to reveal co-immunoprecipitation of endogenous IKK $\alpha$. (g) Like (f), but using THP1 NSC and shDDX3 cells stimulated for $1 \mathrm{~h}$ with CL075 for the co-immunoprecipitation. All blots in Figure 1a-e are representative of at least three independent experiments. Blots in Figure 1f,g are representative of two independent repeat experiments. 
appears to be different from that for IKKe, which we previously determined to lie between aa 100 and 110 of DDX3 [3].

Finally, we wanted to ascertain that endogenous DDX3 and IKK $\alpha$ interact following activation of a relevant immune signalling pathway. Thus, we immunoprecipitated endogenous DDX3 from CL075-stimulated HEK293_TLR7 cells, using a matching isotype antibody as a control for specificity. We observed co-immunoprecipitation of endogenous IKK $\alpha 45 \mathrm{~min}$ after CL075 stimulation (Figure 1e), while the isotype control antibody did not pull down any IKK $\alpha$. In addition, we also carried out a similar co-immunoprecipitation in stable HEK293_TLR7 cell lines that we engineered to express an inducible DDX3 shRNA (short hairpin RNA) construct or an NSC shRNA construct. DDX3 knockdown was induced by treating cells with doxycycline for $48 \mathrm{~h}$, before stimulating cells with CL075 for different time periods, and immunoprecipitating endogenous DDX3 from resulting cell lysates. In this experiment, we again saw endogenous IKK $\alpha$ co-immunoprecipitating with DDX3 45 min after CL075 stimulation in control (NSC) cells, while no IKK $\alpha$ was pulled down with the DDX3 antibody in cells depleted for DDX3 (DDX3 shRNA cells), indicating that the observed DDX3-IKK $\alpha$ interaction bands in NSC cells are specific (Figure 1f). The interaction between DDX3 and IKK $\alpha$ was still detected $90 \mathrm{~min}$ after stimulation, but had disappeared at the $4 \mathrm{~h}$ time point (Figure 1f). These data confirm that endogenous DDX3 and IKK $\alpha$ interact after physiological activation of the TLR7 pathway. Finally, we also immunoprecipitated DDX3 from CL075-stimulated THP1 cells that either expressed an NSC shRNA or the DDX3 shRNA. As THP1s are a human monocyte/macrophage cell line, they likely respond to the TLR7/8 ligand CL075 through TLR8. Again, we observed co-immunoprecipitation of IKK $\alpha$ with DDX3 in CL075-stimulated THP1 cells, but not in unstimulated cells (Figure 1g), indicating that this function is shared between TLR7 and TLR8. There was no pulldown of IKK $\alpha$ in DDX3 knockdown cells, confirming that the interaction is specific.

\section{DDX3 enhances IKKa activation}

Seeing as we previously demonstrated that DDX3 enhances IKKe autophosphorylation [3], we next investigated whether DDX3 has a similar effect on IKK $\alpha$ activation. In radioactive kinase assays with commercially obtained GST-IKK $\alpha$, we detected an IKK $\alpha$ autophosphorylation band that was reproducibly enhanced in the presence of recombinant purified DDX3. The N-terminal domain (aa 1-408) of DDX3 was sufficient to mediate this effect (Figure 2a). Phosphorylation of IKK $\alpha$ at serines 176 and 180 in the activation loop of the kinase is required for and indicative of its activation. To confirm the effect of DDX3 on IKK $\alpha$ phosphorylation and clarify whether it enhances IKK $\alpha$ phosphorylation at these relevant serine residues, we conducted kinase assays with unlabelled ATP followed by detection of phospho-IKK $\alpha$ in WBs with an antibody specific for IKK $\alpha$ phosphorylated at serines $176 / 180$. IKK $\alpha$ phosphorylation at these residues was indeed enhanced in the presence of recombinant DDX3 (aa 1-408) (Figure 2b). Total recombinant IKK $\alpha$ was also detected in this experiment with an anti-IKK $\alpha$ antibody. We suspect that the total IKK $\alpha$ band appears thicker due to the appearance of phosphorylated higher-migrating forms of IKK $\alpha$ rather than uneven loading, as we repeated this experiment many times with reproducible results (Figure 2b). Next, we co-expressed Flag-IKK $\alpha$ and either DDX3 or the vaccinia virus (VACV) protein K7, which antagonises DDX3 [4], in HEK293T cells, lysed the cells after $24 \mathrm{~h}$, and probed for phosphorylated IKK $\alpha$ (Ser176/180) in WBs. As shown in Figure 2c, ectopic expression of DDX3 (aa 1-408) strongly enhanced IKK $\alpha$ phosphorylation, while expression of its VACV antagonist K7 reduced levels of phosphorylated IKK $\alpha$. This demonstrated that the effect of DDX3 on IKK $\alpha$ autophosphorylation also occurs in cells. Finally, we wanted to confirm that endogenous DDX3 is involved in mediating IKK $\alpha$ phosphorylation following activation of a relevant immune signalling pathway. To this end, we used the HEK293_TLR7 cell lines expressing DDX3 shRNA or a NSC. Knockdown of DDX3 was induced for $48 \mathrm{~h}$ before stimulation of the cells with the TLR7 agonist CL075. While there was some background activation present in unstimulated cells,

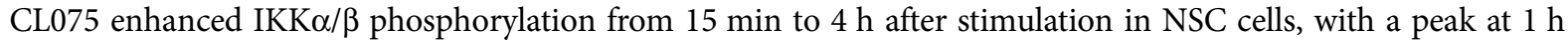
post stimulation. In cells with shRNA-mediated DDX3 knockdown, IKK $\alpha / \beta$ phosphorylation was clearly reduced, with some residual activation detectable at the $2 \mathrm{~h}$ and $4 \mathrm{~h}$ time points (Figure $2 \mathrm{~d}$ ). It should be noted that the antibody for phospho-IKK $\alpha$ is unable to differentiate between phospho-IKK $\alpha$ and phospho-IKK $\beta$ due to conservation of their activation loops, and thus it is possible that phosphorylation of both kinases is detected and affected by DDX3 knockdown in this experiment. However, the fact that we see such a clear reduction with DDX3 knockdown despite a possible contribution of IKK $\beta$ phosphorylation to the overall signal rather supports our conclusion that DDX3 has a marked role in this pathway. 
a

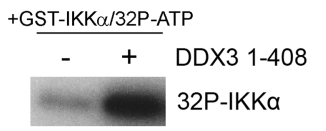

c

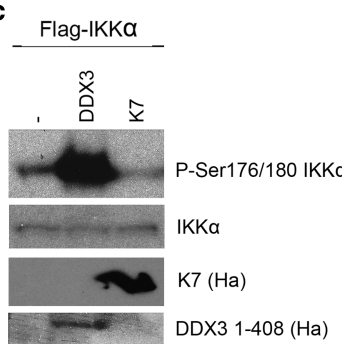

b

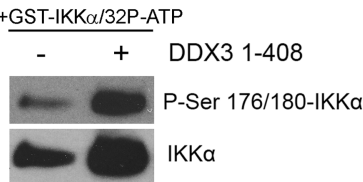

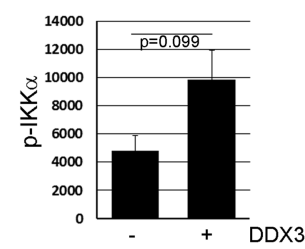

d

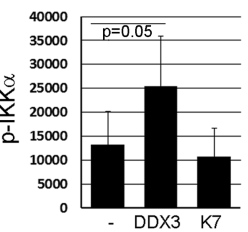

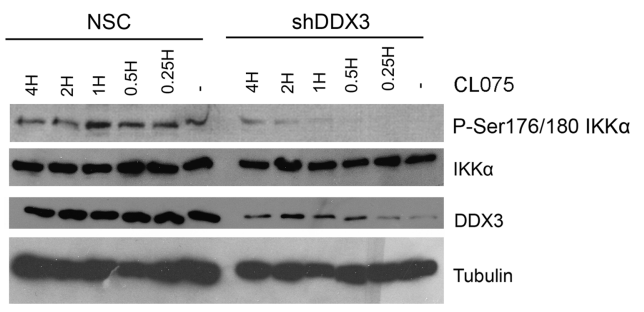

Figure 2. DDX3 enhances autophosphorylation of IKKa in the activation loop.

$(\mathbf{a}, \mathbf{b})$ Kinase assays were carried out with recombinant GST-IKK $\alpha$ (Millipore) in the presence or absence of the N-terminus of DDX3 (aa 1-408), demonstrating that DDX3 enhances autophosphorylation of IKK $\alpha$ in in vitro assays. In (a), the assay was carried out in the presence of [ $\gamma$-32P]-ATP and (auto-)phosphorylated IKK $\alpha$ was detected by SDS-PAGE and autoradiography, which demonstrates incorporation of $[\gamma-32 P]-A T P . \ln (\mathbf{b})$, the assay was carried out in the presence of unlabelled ATP, and IKK $\alpha$ phosphorylated in the activation loop (Ser176/180) was detected using a phospho-specific antibody in WB analysis. Intensity of the phospho-IKK $\alpha$ bands was quantified using ImageJ in three repeat experiments and the graph depicts the average intensities \pm SD. Statistical analysis was carried out using a paired $t$-test. For (c), IKK $\alpha$ was ectopically expressed in HEK293T cells, together with either Ha-DDX3 (1-408) or Ha-K7 (a viral antagonist of DDX3). $24 \mathrm{~h}$ later, cells were harvested, lysed, and subjected to SDS-PAGE and WB analysis to detect IKK $\alpha$ phosphorylated at Ser 176/180, demonstrating that DDX3 also enhances IKK $\alpha$ (auto-)phosphorylation in cells. Intensity of the phospho-IKK $\alpha$ bands was quantified using ImageJ in four repeat experiments and the graph depicts the average intensities $\pm \mathrm{SD}$. Statistical analysis was carried out using a paired $t$-test. For (d), HEK293_TLR7 cells stably transduced with either an inducible shRNA construct for DDX3 (shDDX3) or a corresponding NSC plasmid were treated with doxycycline for $48 \mathrm{~h}$ to induce shRNA expression and then stimulated for the indicated time periods with the TLR7/8 ligand CL075. Cells were then lysed and subjected to SDS-PAGE and Western Blotting to detect IKK $\alpha$ phosphorylated at Ser176/180 and confirm knockdown of DDX3. This experiment demonstrated reduced IKK $\alpha$ phosphorylation in cells with DDX3 knockdown, confirming a role for endogenous DDX3 in IKK $\alpha$ activation downstream of TLR7. All blots in Figure 2 are representative of at least three independent experiments.

\section{DDX3 enhances NIK/IKK $\alpha$-mediated IRF7 activation}

We next hypothesised that increased IKK $\alpha$ activation should result in increased phosphorylation of its substrates. As mentioned in the introduction, we were particularly interested in determining whether DDX3 can regulate IFN induction pathways that are independent of IKKe/TBK1, such as TLR7-induced production of IFN $\alpha$. We therefore investigated whether DDX3 affects NIK/IKK $\alpha$-mediated phosphorylation of IRF7, the main transcription factor required for activation of IFN $\alpha$ promoters. IRF7 is not constitutively expressed in cells other than pDCs and was undetectable by Western blotting in HEK293 cells. In all experiments involving IRF7, we therefore ectopically expressed a small amount of Flag-IRF7. We first overexpressed NIK, IKK $\alpha$, IRF7 and DDX3 in HEK293T cells, followed by detection of phospho-IRF7 by Western blotting (using an antibodyspecific IRF7 phosphorylated at serines 471/472, which is indicative of its activation). As expected, co-expression of NIK and IKK $\alpha$ led to activatory phosphorylation of IRF7 (Figure 3a, lane 2), which was further enhanced by DDX3 expression (Figure 3a, lane 3). To confirm that endogenous DDX3 is required for mediating NIK/IKK $\alpha$-induced IRF7 activation, we next used stable HEK293 cell lines containing the inducible DDX3 shRNA construct or an NSC construct, as described above. We induced knockdown of DDX3 for $48 \mathrm{~h}$, followed by transfection of cells with NIK, IKK $\alpha$, and IRF7 expression constructs. Phosphorylation of IRF7 was significantly reduced in cells with DDX3 knockdown compared with control (NSC) cells (Figure 3b), confirming that DDX3 is an upstream regulator of IRF7 activation by NIK and IKK $\alpha$. 
a
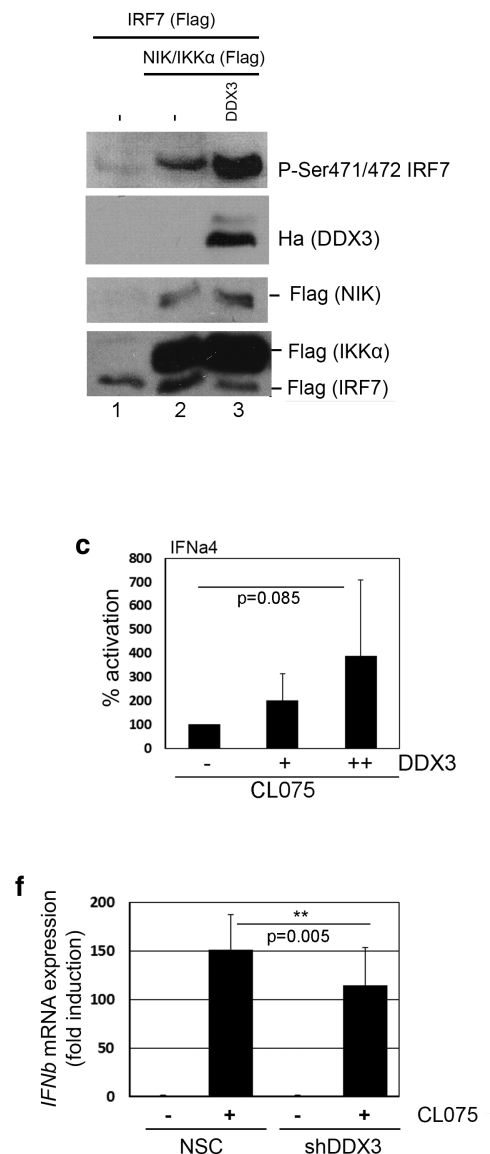

b
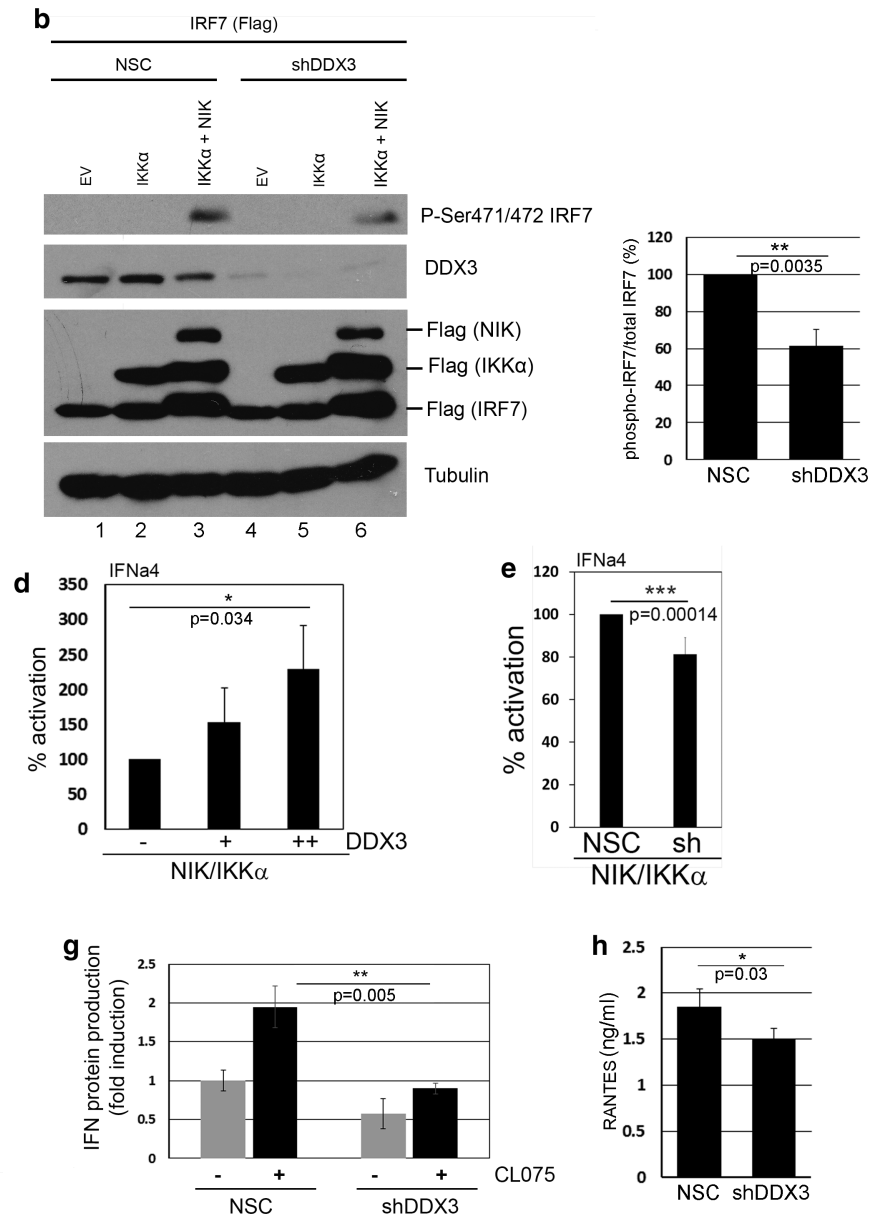

Figure 3. DDX3 regulates IKKa-mediated IRF7 phosphorylation and interferon induction.

Part 1 of 2 (a) HEK293T cells were transfected with expression constructs for Flag-IRF7, Flag-NIK, Flag-IKK $\alpha$, and Ha-DDX3 as indicated, and harvested and lysed for SDS-PAGE and WB analysis $24 \mathrm{~h}$ later. Activation of IRF7 was detected with a phospho-specific antibody for IRF7 phosphorylated at Ser471/472, and expression of NIK, IKK $\alpha$, IRF7, and DDX3 was confirmed using epitope-tag antibodies as indicated. Ectopic expression of DDX3 enhanced NIK/IKK $\alpha$-mediated IRF7 phosphorylation in this setting. (b) HEK293 cells stably transduced with either the inducible DDX3 shRNA construct (shDDX3) or the NSC construct were treated with doxycycline for $24 \mathrm{~h}$ to induce shRNA expression, before being transfected with expression constructs for Flag-IRF7, and empty vector (EV), or expression constructs for Flag-IKK $\alpha$ alone or together with Flag-NIK. Cells were harvested, lysed, and subjected to SDS-PAGE and WB analysis $24 \mathrm{~h}$ after transfection. Activation of IRF7 was detected with the antibody against phospho-IRF7 (Ser471/472), and correct expression of NIK, IKK $\alpha$, and IRF7 was confirmed with the anti-Flag antibody, and knockdown of endogenous DDX3 by blotting for DDX3. We quantified band intensities across four repeat experiments using ImageJ and normalised intensity of phospho-IRF7 to total IRF7. The level of phospho-IRF7 in NIK/ IKK $\alpha$-transfected NSC cells (lane 3) was set to $100 \%$ in each individual experiment and the level of phosphor-IRF7 in NIK/ IKK $\alpha$-transfected shDDX3 cells (lane 6) expressed as a percentage thereof. The graph depicts the mean percentage \pm SD. Statistical analysis was carried out using a paired Student's $t$-test. Cells with DDX3 knockdown (shDDX3) had reduced levels of phospho-IRF7 compared with control (NSC) cells, confirming that endogenous DDX3 is involved in mediating IRF7 phosphorylation downstream of NIK/IKK $\alpha$. (c-e) Cells were transfected with an IFN $\alpha 4$ promoter Firefly luciferase reporter construct together with a pGL3_Renilla luciferase reporter to control for transfection efficiency, and expression constructs as indicated. The Firefly/Renilla ratio was calculated for each sample and then normalised to the unstimulated control. (c) HEK293-TLR7 cells were transfected with reporter constructs and an expression construct for DDX3, and stimulated with CL075 $24 \mathrm{~h}$ after transfection for an additional $24 \mathrm{~h}$. CL075-induced activation was set at $100 \%$ for each individual experiment and all other values calculated relative to this. Data shown represent the mean values from three independent experiments \pm SD. Statistical analysis was carried out using a paired $t$-test. (d) IFN 44 reporter assays were carried out in HEK293 cells transfected with expression constructs for $\mathrm{NIK}, \mathrm{IKK} \alpha$, and DDX3 as indicated. Data analysis and representation as in (c), with 
$\mathrm{NIK} / \mathrm{IKK} \alpha$-induced activation set at $100 \%$. (e) IFN $\alpha 4$ reporter assays were carried out in HEK293 NSC and shDDX3 cells. Knockdown of DDX3 was induced with doxycycline for $48 \mathrm{~h}$ before transfection of cells with reporter constructs and expression constructs for NIK and IKK $\alpha$. Data analysis and representation as in (d), but data represent the mean of nine independent experiments \pm SD. (f) THP1 cells stably transduced with either the inducible DDX3 shRNA construct (shDDX3) or the NSC construct were treated with doxycycline for $48 \mathrm{~h}$ to induce shRNA expression, differentiated with PMA, and then stimulated with TLR7/8 agonist CL075 for $6 \mathrm{~h}$. ifnb mRNA expression was measured using quantitative real-time PCR and is displayed as fold induction over unstimulated control (NSC) cells. Displayed is an average of three experiments \pm SD. Statistical analysis was carried out using a paired $t$-test, with $P$-values indicated in the diagram. (g) Supernatants from experiments with CL075-stimulated THP1 cells from (f) were analysed for type I IFN protein production using HEK-Blue ${ }^{\mathrm{TM}}$ IFN $\alpha / \beta$ sensor cells, based on IFNAR-induced activation of an SEAP reporter. Data are displayed as fold induction over unstimulated control (NSC) cells and as an average of three experiments \pm SD. Statistical analysis was carried out using a paired $t$-test, with $P$-values indicated in the diagram. (h) THP1 NSC or shDDX3 cells were treated with doxycycline for $48 \mathrm{~h}$ to induce shRNA expression and then stimulated with CL075 for $20 \mathrm{~h}$. RANTES cytokine levels were measured with a commercially available ELISA kit $(R \& D)$. The graph depicts the average cytokine levels from four independent experiments $\pm S D$. Statistical analysis was carried out using a paired $t$-test, with $P$-value indicated in the diagram.

\section{DDX3 is part of the TLR7/8 signalling pathway to IFN induction}

IRF7 activation leads to induction of type I interferon promoters. We therefore investigated next whether DDX3 overexpression or knockdown modulates IFN induction downstream of TLR7/8 stimulation and NIK/ IKK $\alpha$. We first used an IFN $\alpha 4$ promoter luciferase construct, which is predominantly activated by IRF7 (rather than IRF3) [20]. Stimulation of HEK293_TLR7 cells with CL075 activated the IFN 44 promoter, and this activation was further enhanced by ectopic expression of increasing amounts of DDX3 (Figure 3c). While this did not reach statistical significance $(P=0.085, n=3$, paired $t$-test), there was nonetheless a trend towards enhanced IFN $\alpha 4$ promoter activation in DDX3-overexpressing cells. We next expressed NIK and IKK $\alpha$ together in HEK293 cells, which - as expected - also strongly activated the IFN $\alpha 4$ promoter. Co-expression of DDX3 significantly enhanced IFN $\alpha 4$ promoter activation induced by $\mathrm{NIK} / \mathrm{IKK} \alpha(P=0.034, n=3$, paired $t$-test), suggesting that it might act downstream of or at the level of these kinases (Figure $3 \mathrm{~d}$ ). We confirmed this further by conducting NIK/IKK $\alpha$-induced IFN $\alpha 4$ reporter gene assays after knocking down expression of endogenous DDX3 in our stable inducible DDX3 shRNA HEK293 cell line. When endogenous DDX3 was knocked down in shDDX3 cells, IFN $\alpha 4$ promoter activation induced by NIK and IKK $\alpha$ was significantly reduced compared with control (NSC) cells ( $P=0.00014, n=9$, paired $t$-test), suggesting that DDX3 acts downstream of the NIK/ IKK $\alpha$ kinase complex in IFN $\alpha 4$ promoter activation (Figure $3 \mathrm{e}$ ).

We next used the THP1 cell line expressing either DDX3 shRNA or the corresponding NSC construct, in order to analyse effects of DDX3 knockdown in cells that endogenously express TLR8. Following induction of DDX3 knockdown, THP1 cells were differentiated with PMA and then stimulated with CL075 for $24 \mathrm{~h}$. Ifnb mRNA levels were up-regulated by CL075, as detected by real-time PCR, and were significantly reduced in DDX3 knockdown (sh) cells compared with control (NSC) cells (Figure 3f) $(P=0.005, n=3$, paired $t$-test). We also measured type I IFN and RANTES protein levels in the supernatant using the HEK-Blue ${ }^{\mathrm{TM}}$ IFN $\alpha / \beta$ reporter cell line and RANTES ELISAs, respectively. IFN protein levels were significantly reduced in DDX3 knockdown (sh) cells compared with control (NSC) cells (Figure 3g) $(P=0.005, n=3$, paired $t$-test). RANTES levels were also significantly reduced in CL075-stimulated DDX3 knockdown (shDDX3) THP1 cells compared with control (NSC) cells. These results confirm that endogenous DDX3 is involved in the signalling pathway leading to IFN induction downstream of TLR7/8.

\section{DDX3 regulates $\mathrm{p} 100$ processing and affects expression of p52-target genes}

Another immune-relevant signalling pathway that relies on the interplay between NIK and IKK $\alpha$ is the alternative NF- $\kappa$ B pathway, which leads to processing of p100 to p52 [16]. We therefore investigated whether DDX3 also regulates this separate IKK $\alpha$-dependent signalling pathway. First, we expressed and stained Ha-p100 in HEK293T cells in order to examine its subcellular localisation (both p100 and the processed p52 carry the Ha-tag when expressed from our expression construct and will therefore be visualised by our 
immunofluorescent staining approach). Co-expression of NIK/IKK $\alpha$ with Ha-p100 increased the nuclear localisation of $\mathrm{p} 100 / \mathrm{p} 52$ as expected (due to processing of $\mathrm{p} 100$ to $\mathrm{p} 52$, which can then translocate into the nucleus). Additional co-expression of DDX3 led to a further increase in nuclear p52, suggesting that DDX3 can promote p100 processing to p52 (Figure 4a). Next, we used WB analysis to confirm that DDX3 affects p100 processing. We induced knockdown of DDX3 in our stably transduced HEK293 DDX3 shRNA (or NSC) cells by treating them with doxycycline for $48 \mathrm{~h}$, and then ectopically expressed NIK and IKK $\alpha$, before harvesting cells for WB analysis. NIK and IKK $\alpha$ co-expression clearly induced p100 phosphorylation and subsequent degradation to p52 in control (NSC) cells (Figure 4b, lane 3), while p100 phosphorylation was greatly reduced and p52 levels were lower in DDX3 knockdown (shDDX3) cells (Figure 4b, compare lane 3 and lane 6 and see quantification graphs). We then proceeded to show that DDX3 knockdown affects downstream gene expression mediated by p52 and chose the well-established p52-target gene BAFF (B-cell activation factor) as our read-out [16]. We again used the THP1 cell lines stably transduced with either the DDX3 shRNA plasmid or an NSC
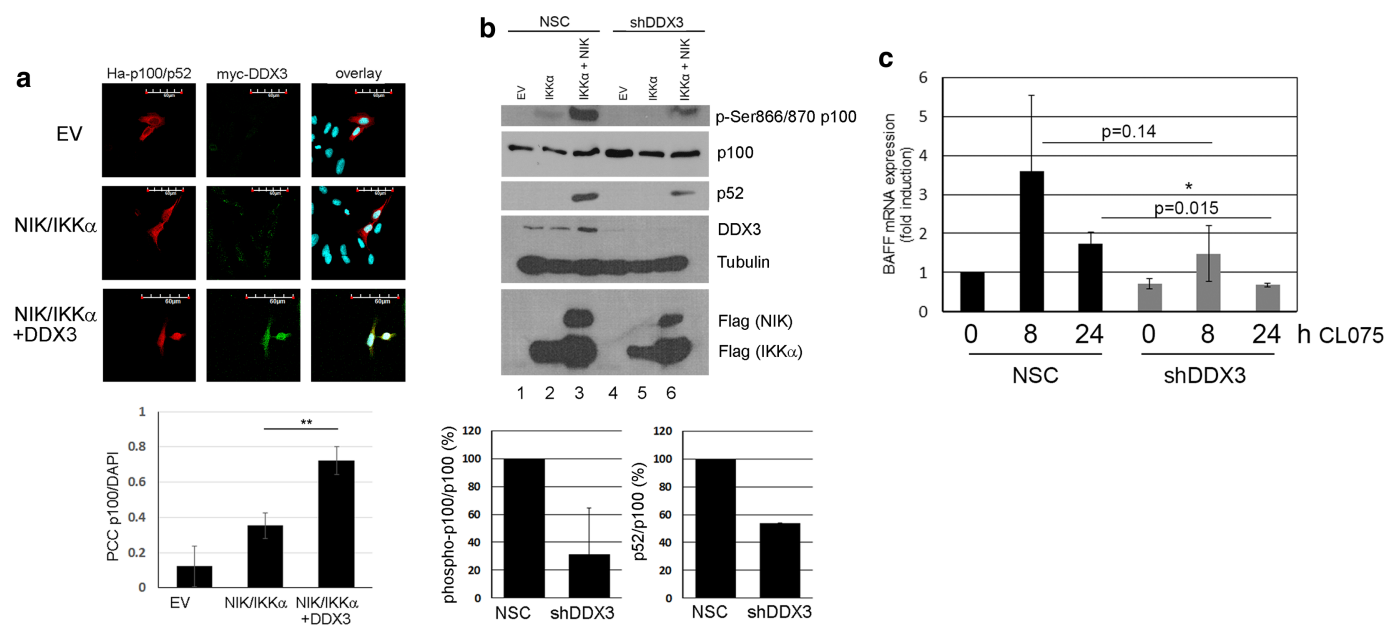

Figure 4. DDX3 regulates p100 processing and expression of a p52-target gene.

(a) HEK293 cells were cotransfected with expression constructs for Ha-p100, Flag-IKK $\alpha$, Flag-NIK, and myc-DDX3 as indicated. p100 and processed p52 were detected with an antibody against the Ha-tag and DDX3 was detected with an antibody against the myc-tag, as well as species-specific secondary antibodies (AlexaFluor488/green for DDX3 and AlexaFluor594/red for p100/p52). For quantifying nuclear localisation of p52, 30 transfected cells were selected for each condition and the PCC between AlexaFluor594 (p100/p52) and the DAPI nuclear stain was. The graph shows the mean PCC \pm SD for each condition. Statistical analysis was carried out using an unpaired Student's $t$-test $\left({ }^{* \star} P \leq 0.01\right)$. (b) HEK293 cells stably transduced with either the inducible DDX3 shRNA construct (shDDX3) or the NSC construct were treated with doxycycline for $24 \mathrm{~h}$ to induce shRNA expression, before being transfected with empty vector (EV), or expression constructs for Flag-IKK $\alpha$ alone or together with Flag-NIK. Cells were harvested, lysed, and subjected to SDS-PAGE and WB analysis $24 \mathrm{~h}$ after transfection. WBs were probed with a phospho-specific antibody for p100 phosphorylated at Ser866/870 (the phosphorylation event that precedes degradation), an antibody that detects both p100 and the processed p52 (second and third panel), a DDX3 antibody to confirm knockdown, a tubulin antibody to confirm equal loading, and a Flag antibody to confirm expression of both Flag-IKK $\alpha$ and Flag-NIK. We quantified band intensities across two repeat experiments using ImageJ and normalised intensity of phospho-p100 (left graph) or p52 bands (right graph) to total p100 bands. The level of phospho-p100 or p52 in NIK/IKK $\alpha$-transfected NSC cells (lane 3) was set to $100 \%$ in each individual experiment and the level of phospho-p100 or p52 in NIK/IKK $\alpha$-transfected shDDX3 cells (lane 6) expressed as a percentage thereof. The graphs depict the mean percentage \pm SD. Phosphorylation of p100 and p100 processing to p52 were reduced in DDX3 knockdown cells (shDDX3) compared with control cells (NSC). Blots shown are representative of two independent repeat experiments. (c) THP1 cells stably transduced with either the inducible DDX3 shRNA construct (shDDX3) or the NSC construct were treated with doxycycline for $48 \mathrm{~h}$ to induce shRNA expression, differentiated with PMA, and then stimulated with TLR7/8 agonist CL075 for the indicated time periods. BAFF mRNA expression was measured using quantitative real-time PCR and is displayed as fold induction over unstimulated control (NSC) cells. Displayed is an average of three experiments \pm SD. Statistical analysis was carried out using a paired $t$-test, with $P$-values indicated in the diagram. 
plasmid, and induced knockdown by treating cells for $48 \mathrm{~h}$ with doxycycline. We then differentiated THP1 cells with PMA for $24 \mathrm{~h}$ before stimulation with CL075. We observed up-regulation of BAFF mRNA expression (measured by real-time PCR) at 8 or $24 \mathrm{~h}$ in NSC cells, which was reduced in DDX3 knockdown (shDDX3) cells (Figure 4c), reaching statistical significance for the $24 \mathrm{~h}$ time point $(P=0.015, n=3$, paired $t$-test). This data demonstrate that DDX3 is also involved in mediating alternative NF- $\mathrm{KB}$ activation downstream of NIK/ IKK $\alpha$ in the TLR7/8 pathway and thus broadens the downstream effects of DDX3 modulation beyond IFN-inducing pathways.

\section{Discussion}

Our study demonstrates that DDX3 directly interacts with IKK $\alpha$ and enhances its kinase activity, thereby regulating the activity of IKK $\alpha$-dependent downstream signalling pathways. We had previously shown that DDX3 interacts with IKKe and facilitates its autophosphorylation and auto-activation [3]. According to our new data, DDX3 exerts a similar effect on the related kinase IKK $\alpha$ and can thereby contribute to additional IKKe/ TBK1-independent innate immune signalling pathways. A very interesting study published while this project was ongoing also demonstrated the DDX3-IKK $\alpha$ interaction, in that case occurring during HCV infection or transfection of poly(I:C) [21]. The study showed that DDX3 co-localised with the HCV $3^{\prime} \mathrm{UTR}$ RNA and IKK $\alpha$. Interestingly, the DDX3-IKK $\alpha$ interaction in this paper was suggested to result from direct recognition of HCV RNA by DDX3 [21], thus indicating that DDX3 acts as a viral sensor in this context. This is in line with a study published by Oshiumi et al. [7], which suggested that DDX3 recognises HCV RNA and then signals via RIG-I/MDA5/MAVS to IFN induction. However, our previous studies have characterised DDX3 as a downstream signalling adaptor linking IKKe and the transcription factor IRF3 [3,4], which is also supported by several other studies $[5,6,8,9]$. In this present study, we observed the DDX3-IKK $\alpha$ interaction in the absence of viral RNA, namely following stimulation of the TLR7/8 pathway with the synthetic ligand CL075, a thiazoloquinolone derivative. Knockdown of DDX3 also affected signals downstream of NIK/IKK $\alpha$, suggesting that $\mathrm{DDX} 3$ is able to exert an equivalent role as a signalling adaptor also in $\mathrm{NIK} / \mathrm{IKK} \alpha$-dependent pathways. $\mathrm{Li}$ et al.'s paper [21] demonstrated that IKK $\alpha$ was required for SREBP-mediated lipogenesis and lipid droplet formation in HCV-infected Hu7.5.1 cells. As lipid droplets are crucial for efficient HCV replication and assembly, IKK $\alpha$ knockdown strongly suppressed HCV replication in these cells. DDX3 is also required for HCV replication $[22,23]$ and it is therefore conceivable that enhancement of IKK $\alpha$ signalling contributes to DDX3's proviral role in HCV replication. Our study complements this work by demonstrating that the interaction between DDX3 and IKK $\alpha$ is direct and promotes IKK $\alpha$ autophosphorylation and -activation, resulting in enhanced phosphorylation of IKKo's downstream targets. While Li et al. [21] focussed on SREBP expression mediated by CBP, we demonstrate that IRF7 activation and p100 phosphorylation and processing are also affected by DDX3's regulation of IKK $\alpha$. Our work therefore suggests that the DDX3-IKK $\alpha$ link might be a double-edged sword for the virus. On the one hand, it promotes lipid droplet formation, which favours the virus [21], but on the other hand it promotes production of interferons and pro-inflammatory cytokines (this study). In this context, it is very interesting that, in a follow-up study to their original work, the Liang laboratory showed that the DDX3-IKK $\alpha$ interaction appears to be dynamically regulated during HCV infection, with it only occurring early during infection. When viral protein production was high, DDX3 clustered with the HCV core protein on lipid droplets instead [24].

Our work shows that IKK $\alpha$ is another kinase whose activity can be regulated directly by DDX3, similar to

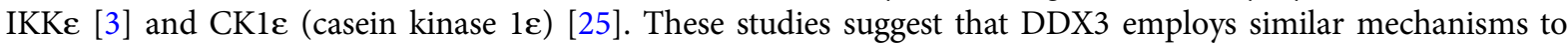
facilitate activation of different kinases and mediate phosphorylation of their downstream targets. Interestingly though, the C-terminal region of DDX3 was required and sufficient for CK1 activation [25], whereas the $\mathrm{N}$-terminus was required and sufficient for IKKe and IKK $\alpha$ activation. We showed here that, despite the relatedness of the IKKs, IKK $\alpha$ interacts with DDX3 in a slightly different manner compared with IKKe. While IKKe binds to N-terminal and C-terminal regions of DDX3 (aa 1-110 and 409-662), but not to DDX3 (aa 139-408) [3], IKK $\alpha$ still binds to DDX3 (aa 139-408) but fails to bind to the C-terminus of DDX3 (aa 409$662)$.

Importantly, the finding that DDX3 can directly regulate IKK $\alpha$ greatly broadens the downstream effects of DDX3 manipulation on innate immune responses: (1) it affects TBK1/IKKe-independent induction of type I interferons downstream of TLR7/8 (and most probably TLR9 given the similarity of the two signalling pathways) and (2. it affects IKK $\alpha$-mediated signalling pathways that are independent of IRF3/7, such as the alternative NF- $\mathrm{KB}$ pathway, and thereby regulates expression of additional sets of genes beyond type I interferons. 
These findings, in combination with the work published by the Liang laboratory $[21,24]$ that showed an effect on the CBP-SREBP pathway, suggest that DDX3 generally affects downstream signalling pathways emanating from IKK $\alpha$. It is not unlikely that additional downstream pathways, such as the conventional NF-KB pathway, could also be affected by DDX3's regulation of IKK $\alpha$ [26].

Its relevance for physiological processes regulated by these pathways is worth investigating in future studies. For example, by affecting the alternative NF- $\mathrm{KB}$ pathway, DDX3 could be involved in CD40 signalling, development of secondary lymphoid organs, and B-cell survival and maturation of B-cells [17]. While it remains to be confirmed that our finding of DDX3 regulating TLR7-mediated IFN induction extends to pDCs, the physiologically relevant cell type for this signalling pathway, this could potentially indicate a link between DDX3 and systemic lupus erythematosus (SLE). TLR7 signalling is strongly linked SLE pathogenesis, with TLR7 activation being triggered by anti-RNP immune complexes, resulting in the production of large amounts of type I IFN from pDCs [27-29]. SLE is an autoimmune condition with a much higher prevalence in women compared with men, and interestingly, DDX3(X) escapes X-inactivation [30] and might indeed be expressed to higher levels in females compared with males [31-33]. Furthermore, there is also some evidence that the TLR7 pathway is more active in females compared with males [34,35]. Based on our data demonstrating the involvement of DDX3 in TLR7 signalling, higher expression of DDX3 in females could conceivably contribute to increased TLR7 pathway activity and thereby to the increased female prevalence of SLE.

\section{Abbreviations \\ aa, amino acid; BAFF, B-cell activation factor; CK, casein kinase; DDX, DEAD-box; GST, Glutathione-S-Transferase; HEK, human embryonic kidney; IFN, interferon; IKK, IKB kinase; IP, immunoprecipitation; IRF, interferon regulatory factor; MAVS, mitochondrial antiviral signalling; mt, mutant; NF-кB, Nuclear Factor-кB; Ni-Ag, Nickel-Agarose; NIK, NF-кB-inducing kinase; NSC, non-silencing control; PCC, Pearson's Correlation Coefficient; pDC, plasmacytoid dendritic cell; PRR, pattern recognition receptor; RIG, retinoic acid-inducible gene; SD, standard deviation; shRNA, short hairpin RNA; SLE, systemic lupus erythematosus; TBK, TANK-binding kinase; TLR, Toll-like receptor; VACV, vaccinia virus; WB, Western blot; wt, wild type.}

\section{Author Contribution}

A.F., L.G., and Y.H. designed and carried out experiments and analysed data. M.S. devised the hypothesis and experimental strategy, guided experimental design, and analysed data. A.F. and M.S. co-wrote the paper and all authors checked the manuscript before publication.

\section{Acknowledgements}

We gratefully acknowledge support of this research through funding from Science Foundation Ireland [09/RFP/ BIC2188] and the Irish Health Research Board [HRA/2009/171 and HRA_POR/2011/58].

\section{Competing Interests}

The Authors declare that there are no competing interests associated with the manuscript.

\section{References}

1 Fullam, A. and Schröder, M. (2013) DExd/H-box RNA helicases as mediators of anti-viral innate immunity and essential host factors for viral replication. Biochim. Biophys. Acta 1829, 854-865 https://doi.org/10.1016/j.bbagrm.2013.03.012

2 Gu, L., Fullam, A., McCormack, N., Höhn, Y. and Schröder, M. (2017) DDX3 directly regulates TRAF3 ubiquitination and acts as a scaffold to co-ordinate assembly of signalling complexes downstream from MAVS. Biochem. J. 474, 571-587 https://doi.org/10.1042/BCJ20160956

$3 \mathrm{Gu}$, L., Fullam, A., Brennan, R. and Schröder, M. (2013) The human DEAD-box helicase 3 couples IKK-epsilon to IRF3 activation. Mol. Cell. Biol. 33, 2004-2015 https://doi.org/10.1128/MCB.01603-12

4 Schröder, M., Baran, M. and Bowie, A.G. (2008) Viral targeting of DEAD box protein 3 reveals its role in TBK1//KK-epsilon-mediated IRF activation. EMBO J. 27, 2147-2157 https://doi.org/10.1038/emboj.2008.143

5 Yu, S., Chen, J., Wu, M., Chen, H., Kato, N. and Yuan, Z. (2010) Hepatitis B virus polymerase inhibits RIG-I- and Toll-like receptor 3-mediated beta interferon induction in human hepatocytes through interference with interferon regulatory factor 3 activation and dampening of the interaction between TBK1/IKK\{epsilon\} and DDX3. J. Gen. Virol. 91, 2080-2090 https://doi.org/10.1099/vir.0.020552-0

6 Wang, H. and Ryu, W.-S. (2010) Hepatitis B virus polymerase blocks pattern recognition receptor signaling via interaction with DDX3: implications for immune evasion. PLos Pathog. 6, e1000986 https://doi.org/10.1371/journal.ppat.1000986

7 Oshiumi, H., Sakai, K., Matsumoto, M. and Seya, T. (2010) DEAD/h BOX 3 (DDX3) helicase binds the RIG-I adaptor IPS-1 to up-regulate IFN-beta-inducing potential. Eur. J. Immunol. 40, 940-948 https://doi.org/10.1002/eji.200940203 
8 DeFilippis, V.R., Alvarado, D., Sali, T., Rothenburg, S. and Fruh, K. (2010) Human cytomegalovirus induces the interferon response via the DNA sensor ZBP1. J. Virol. 84, 585-598 https://doi.org/10.1128/JVI.01748-09

9 Soulat, D., Burckstummer, T., Westermayer, S., Goncalves, A., Bauch, A., Stefanovic, A. et al. (2008) The DEAD-box helicase DDX3X is a critical component of the TANK-binding kinase 1-dependent innate immune response. EMBO J. 27, 2135-2146 https://doi.org/10.1038/emboj.2008.126

10 Pfaller, C.K. and Conzelmann, K.K. (2008) Measles virus V protein is a decoy substrate for IкB kinase $\alpha$ and prevents Toll-like receptor 7/9-mediated interferon induction. J. Virol. 82, 12365-12373 https://doi.org/10.1128/JVI.01321-08

11 Hoshino, K., Sugiyama, T., Matsumoto, M., Tanaka, T., Saito, M., Hemmi, H. et al. (2006) Ikb kinase- $\alpha$ is critical for interferon-alpha production induced by Toll-like receptors 7 and 9. Nature 440, 949-953 https://doi.org/10.1038/nature04641

12 Diebold, S.S., Kaisho, T., Hemmi, H., Akira, S. and Reis e Sousa, C. (2004) Innate antiviral responses by means of TLR7-mediated recognition of single-stranded RNA. Science 303, 1529-1531 https://doi.org/10.1126/science.1093616

13 Wang, R.P., Zhang, M., Li, Y., Diao, F.C., Chen, D., Zhai, Z. et al. (2008) Differential regulation of IKK alpha-mediated activation of IRF3/7 by NIK. Mol. Immunol. 45, 1926-1934 https://doi.org/10.1016/j.molimm.2007.10.034

14 Kawai, T., Sato, S., Ishii, K.J., Coban, C., Hemmi, H., Yamamoto, M. et al. (2004) Interferon-alpha induction through Toll-like receptors involves a direct interaction of IRF7 with MyD88 and TRAF6. Nat. Immunol. 5, 1061-1068 https://doi.org/10.1038/ni1118

15 Liu, Y.-J. (2005) IPC: professional type 1 interferon-producing cells and plasmacytoid dendritic cell precursors. Annu. Rev. Immunol. 23, 275-306 https://doi.org/10.1146/annurev.immunol.23.021704.115633

16 Dejardin, E., Droin, N.M., Delhase, M., Haas, E., Cao, Y., Makris, C. et al. (2002) The lymphotoxin-beta receptor induces different patterns of gene expression via two NF-kB pathways. Immunity 17, 525-535 https://doi.org/10.1016/S1074-7613(02)00423-5

17 Sun, S.C. (2012) The noncanonical NF-кB pathway. Immunol. Rev. 246, 125-140 https://doi.org/10.1111/j.1600-065X.2011.01088.x

18 Rückle, A., Haasbach, E., Julkunen, I., Planz, O., Ehrhardt, C. and Ludwig, S. (2012) The NS1 protein of influenza A virus blocks RIG-I-mediated activation of the noncanonical NF-кB pathway and p52/RelB-dependent gene expression in lung epithelial cells. J. Virol. 86, 10211-7 https://doi.org/ 10.1128/JVI.00323-12

19 Liu, P., Li, K., Garofalo, R.P. and Brasier, A.R. (2008) Respiratory syncytial virus induces RelA release from cytoplasmic 100-kDa NF-kB2 complexes via a novel retinoic acid-inducible gene-l-NF-kB-inducing kinase signaling pathway. J. Biol. Chem. 283, 23169-23178 https://doi.org/10.1074/jbc. M802729200

20 Österlund, P.I., Pietilä, T.E., Veckman, V., Kotenko, S.V. and Julkunen, I. (2007) IFN regulatory factor family members differentially regulate the expression of type III IFN (IFN- $\lambda$ ) genes. J. Immunol. 179, 3434-3442 https://doi.org/10.4049/jimmunol.179.6.3434

21 Li, Q., Pene, V., Krishnamurthy, S., Cha, H. and Liang, T.J. (2013) Hepatitis C virus infection activates an innate pathway involving IKK-alpha in lipogenesis and viral assembly. Nat. Med. 19, 722-729 https://doi.org/10.1038/nm.3190

22 Randall, G., Panis, M., Cooper, J.D., Tellinghuisen, T.L., Sukhodolets, K.E., Pfeffer, S. et al. (2007) Cellular cofactors affecting hepatitis C virus infection and replication. Proc. Natl Acad. Sci. U.S.A. 104, 12884-12889 https://doi.org/10.1073/pnas.0704894104

23 Ariumi, Y., Kuroki, M., Abe, K., Dansako, H., Ikeda, M., Wakita, T. et al. (2007) DDX3 DEAD-box RNA helicase is required for hepatitis C virus RNA replication. J. Virol. 81, 13922-13926 https://doi.org/10.1128/JVI.01517-07

24 Pène, V., Li, Q., Sodroski, C., Hsu, C.-S. and Liang, T.J. (2015) Dynamic interaction of stress granules, DDX3X, and IKK- $\alpha$ mediates multiple functions in hepatitis C virus infection. J. Virol. 89, 5462-5477 https://doi.org/10.1128/JVI.03197-14

25 Cruciat, C.-M., Dolde, C., de Groot, R.E.A., Ohkawara, B., Reinhard, C., Korswagen, H.C. et al. (2013) RNA helicase DDX3 is a regulatory subunit of casein kinase 1 in Wnt- $\beta$-catenin signaling. Science 339, 1436-1441 https://doi.org/10.1126/science.1231499

26 Wang, X., Wang, R., Luo, M., Li, C., Wang, H.X., Huan, C.C. et al. (2017) (DEAD)-box RNA helicase 3 modulates NF-kB signal pathway by controlling the phosphorylation of PP2A-C subunit. Oncotarget 8, 33197-33213 https://doi.org/10.18632/oncotarget.16593

27 Barrat, F.J., Meeker, T., Gregorio, J., Chan, J.H., Uematsu, S., Akira, S. et al. (2005) Nucleic acids of mammalian origin can act as endogenous ligands for Toll-like receptors and may promote systemic lupus erythematosus. J. Exp. Med. 202, 1131-1139 https://doi.org/10.1084/jem.20050914

28 Krieg, A.M. and Vollmer, J. (2007) Toll-like receptors 7, 8, and 9: linking innate immunity to autoimmunity. Immunol. Rev. 220, 251-269 https://doi. org/10.1111/j.1600-065X.2007.00572.x

29 Krieg, A.M. (2007) The toll of too much TLR7. Immunity 27, 695-697 https://doi.org/10.1016/j.immuni.2007.11.001

30 Carrel, L. and Willard, H.F. (2005) X-inactivation profile reveals extensive variability in X-linked gene expression in females. Nature 434, 400-404 https://doi.org/10.1038/nature03479

31 Lopes, A.M., Burgoyne, P.S., Ojarikre, A., Bauer, J., Sargent, C.A., Amorim, A. et al. (2010) Transcriptional changes in response to X chromosome dosage in the mouse: implications for X inactivation and the molecular basis of Turner Syndrome. BMC Genomics 11, 82 https://doi.org/10.1186/ 1471-2164-11-82

32 Chen, C.-Y., Chan, C.-H., Chen, C.-M., Tsai, Y.-S., Tsai, T.-Y., Wu Lee, Y.-H. et al. (2016) Targeted inactivation of murine Ddx3x: essential roles of Ddx3x in placentation and embryogenesis. Hum. Mol. Genet. 25, 2905-2922 https://doi.org/10.1093/hmg/ddw143

33 Yang, X., Schadt, E.E., Wang, S., Wang, H., Arnold, A.P., Ingram-Drake, L. et al. (2006) Tissue-specific expression and regulation of sexually dimorphic genes in mice. Genome Res. 16, 995-1004 https://doi.org/10.1101/gr.5217506

34 Berghofer, B., Frommer, T., Haley, G., Fink, L., Bein, G. and Hackstein, H. (2006) TLR7 ligands induce higher IFN-\{alpha\} production in females. J. Immunol. 177, 2088-2096 https://doi.org/10.4049/jimmunol.177.4.2088

35 Meier, A., Chang, J.J., Chan, E.S., Pollard, R.B., Sidhu, H.K., Kulkarni, S. et al. (2009) Sex differences in the Toll-like receptor-mediated response of plasmacytoid dendritic cells to HIV-1. Nat. Med. 15, 955-959 https://doi.org/10.1038/nm.2004 